\title{
Modelo de gestión estratégica para el posicionamiento comercial en el mercado internacional del cacao Amazonas, Perú
}

\section{Model of strategic management for the commercial positioning in the international market of cocoa Amazonas, Peru}

\author{
Roberto Carlos Mori Zabarburú ${ }^{1}$ Yuri Reina Marín ${ }^{2}$
}

\section{RESUMEN}

Esta investigación propone un modelo de gestión estratégica que permita mejorar el posicionamiento comercial en el mercado internacional del cacao Amazonas Perú, la investigación fue descriptiva propositiva. Se utilizaron datos de encuestas originales en 100 fincas cacaoteras en las provincias de Bagua y Utcubamba en la Región Amazonas, que caracterizaron el cultivo de cacao y las principales variables socioeconómicas. Además se aplicó una entrevista a los gerentes de las organizaciones de productores y al Director de la Mesa Técnica Regional de Cacao con la finalidad de diagnosticar el mercado objetivo. Para el análisis interno y externo se utilizó la matriz FODA. Con estos datos se construyó un vector de crecimiento de oportunidades con ayuda de la Matriz Ansoff. Finalmente, las opciones estratégicas identificadas se sistematizaron en un modelo de gestión. El modelo generado propone estrategias de penetración en los mercados, desarrollo de productos y mercados y diversificación, que coadyuvarán al futuro desarrollo de las organizaciones productoras de cacao.

Palabras clave: Cacao, posicionamiento, gestión estratégica, Amazonas, Perú.

\begin{abstract}
This research proposes a strategic management model that allows improving the commercial positioning in the international cocoa market in Amazonas Peru, the research was descriptive and proactive. Data from original surveys were used in 100 cocoa farms in the provinces of Bagua and Utcubamba in the Amazon Region, which characterized the cultivation of cocoa and the main socioeconomic variables. In addition, an interview was applied to the managers of the producer organizations and to the Director of the Regional Technical Bureau of Cocoa with the purpose of diagnosing the target market. The SWOT matrix was used for the internal and external analysis. With these data, a growth vector of opportunities was built with the help of the Ansoff Matrix. Finally, the strategic options identified were systematized in a management model. The generated model proposes strategies for market penetration, product and market development and diversification, which will contribute to the future development of cocoa producing organizations.
\end{abstract}

Keywords: Cocoa, positioning, strategic management, Amazonas, Peru.

\footnotetext{
Ingeniero agroindustrial, especialista en manejo de cadenas productivas. Correo electrónico: rocamori@gmail.com

${ }^{2}$ Ingeniero Industrial, Profesor auxiliar a Tiempo completo de la Facultad de Ingeniería Zootecnista, Biotecnología y Agronegocios de la Universidad Nacional Toribio Rodríguez de Mendoza de Amazonas; especialista en cadena de suministro. Correo electrónico: yuri.reina@untrm.edu.pe
} 


\section{INTRODUCCIÓN}

El cacao es el tercer producto de exportación agrícola más importante del mundo y el segundo cultivo comercial más importante en los trópicos (Blare \& Useche, 2013). Se estima que más del 80-90\% del cacao es producido por 5-6 millones de pequeñas granjas de cacao (Gayi, 2014) administradas por la familia en más de 50 países en todo el mundo (ECLAC, FAO, \& IICA, 2017). Perú es el cuarto productor del grano en Latinoamérica y el Caribe entre 2006 - 2016 con una producción promedio estimado en 56586 TM, debajo de Brasil, Ecuador y República Dominicana. Más de $65 \%$ de la producción de Perú se realiza en las regiones de Cusco y Ayacucho. En cuanto al segmento del cacao fino de aroma, Perú es el cuarto productor mundial con $9 \%$ de participación mundial. El cacao representa para el país, el sustento de alrededor de 45000 agricultores (Arvelo et al., 2016). La región Amazonas aporta el 4\% de la producción nacional de cacao con una superficie sembrada de 7254 hectáreas, las principales zonas productivas son las provincias de Bagua con $2975.5 \mathrm{TM} / \mathrm{año} y$ Utcubamba con 978.7 TM/año y, existen en la región alrededor de 5500 productores (Rosa-Pérez, Canales, \& Santoyo, 2015). La problemática en torno al cultivo es que, los productores de cacao son en su gran mayoría familiares pobres. Se estima que reciben sólo $6.6 \%$ del pago que realizan los consumidores. El punto a favor, es que a diferencia del cacao convencional, los productores de cacao diferenciado (aromático, fino y orgánico) perciben mejores precios por el producto. En promedio, en los tres años pasados se pagaron por cacao certificado entre 4-20\% más que los precios internacionales en general. Sumado a ello, se ha recibido primas superiores al $23 \%$ encima del precio por cacao fino y aromático, y los precios pagados por cacao fino exclusivo es 66\% (Arvelo et al., 2016). Por ello, la producción de cacao diferenciado es una oportunidad para mejorar la calidad de vida de los productores. Los territorios que sostienen los cultivos de cacao en Amazonas se encuentran vinculadas a las comunidades nativas (Rosa-Pérez et al., 2015), por lo que, el fomento del cacao diferenciado presenta sostenibilidad para la conservación de la biodiversidad y el apoyo a la economía familiar. Por ello, ante la amenaza de una posible caída de precios internacionales de cacao, como lo sucedido en el periodo entre marzo de 2016 a febrero de 2017 (ECLAC et al., 2017), existe una potencialidad de crecimiento con base en la estabilidad en precios que caracteriza a los cacaos diferenciados (Arvelo et al., 2016), y una oportunidad para el desarrollo sostenible. Sin embargo, para este cometido, existe la necesidad de consolidar el posicionamiento de la denominación de origen Cacao Amazonas Perú en el comercio internacional. Por los motivos expuestos, se planteó realizar una investigación descriptiva propositiva para proponer un modelo de gestión estratégica que permita mejorar el posicionamiento comercial en el mercado internacional del cacao Amazonas, Perú.

\section{MATERIAL Y MÉTODO}

\subsection{Diseño de la investigación}

El diseño de investigación es descriptivo propositivo. Esto significa que se utilizaron un conjunto de técnicas y procedimientos que permitieron diagnosticar y proponer lineamientos para la resolución de los problemas identificados. No se administraron tratamientos ni se manipularon variables. La investigación ha permitido proponer un modelo de gestión estratégico para el posicionamiento comercial en el mercado internacional del cacao Amazonas Perú, con base en el análisis propositivo de la situación actual de la producción y comercialización en la provincia de Utcubamba y Bagua.

\subsection{Población, muestra y muestreo}

\section{Población:}

La población la conformaron los socios de las tres cooperativas: Cooperativa Central de Productores Agrarios de Amazonas, Asociación de Productores y Cafetaleros de Amazonas y Cooperativa Cafetalera La Palma Ltda. Que se encuentran enmarcadas dentro del ámbito de la Denominación de Origen.

\section{Muestra:}

Para determinar el tamaño de muestra, se aplicó la siguiente relación estadística:

$$
n=\frac{Z^{2} P Q N}{E^{2}(N-1)+Z^{2} P Q}
$$

Donde:
$\mathbf{N}$ : población
n: tamaño de muestra
Z: 1,96 (al 95\% de confianza)
P: 0,5 nivel de aceptación
Q: 0,5 nivel de fracaso
E: 0,1 error de estimación 
El tamaño de muestra resultó aproximadamente de 90 productores. Sin embargo, se decidió incrementarla a 100 productores. La distribución de las muestras dentro de las organizaciones se realizó en forma proporcional al número de socios de cada una. En ese sentido, la muestra se distribuyó de la siguiente manera: CEPROAA 33 productores, APROCAM 38 productores y Cooperativa Cafetalera La Palma 29 productores.

En la etapa cualitativa, se eligió una muestra no probabilística, conformada por tres unidades de estudio, las cuales están representadas por los gerentes de las tres organizaciones de productores de cacao. Sin embargo, Cooperativa Cafetalera La Palma Ltda. en el año 2016 se desintegró y finiquitó sus actividades, aunque de manera individual sus ex integrantes continúan sus actividades productivas. Por dicho motivo, se consideró como tercera unidad de análisis al director General de PRO Cacao Amazonas. Las tres unidades nos proporcionaron la información productiva, organizacional y comercial para la caracterización cualitativa de la investigación.

\subsection{Método de recolección de datos}

El método que se empleó es el analítico e inductivo, porque a partir de los hechos observados se sistematizaron los resultados y conclusiones de la investigación.

\subsection{Técnicas}

Las técnicas utilizadas fueron: Encuestas, entrevistas, análisis FODA, matriz Ansoff.

\subsection{Instrumentos}

Los instrumentos utilizados para la aplicación de las técnicas de recolección de datos fueron: cuestionario, guía de entrevista y guía para el análisis FODA.

\subsection{Procedimientos}

La investigación se realizó en tres etapas: en la primera se recolectó la información respecto a las variables cuantitativas para caracterizar la producción de cacao en las unidades de estudio. En la segunda etapa se realizó un diagnóstico de las variables cualitativas que explican el estado actual del mercado objetivo y las brechas de calidad con respecto al comercio internacional del cacao. Finalmente, con los insumos obtenidos en la tercera etapa se desarrollaron estrategias con la finalidad de reducir las brechas comerciales y conseguir en el largo plazo el posicionamiento del Cacao Amazonas, Perú.

\subsection{Análisis de datos}

Los datos recolectados a través de la encuesta fueron sistematizados en hojas de cálculo, y con ellos se generaron diagramas de frecuencia para caracterizar la producción de cacao en las provincias de Bagua y Utcubamba. La estadística descriptiva también permitió representar las características económicas, de comercialización y distribución de las unidades productivas. Los datos cualitativos de le entrevista fueron ordenados y analizados, evaluando las similitudes y diferencias en las apreciaciones hechas por los entrevistados. Posteriormente se determinaron en qué preguntas los entrevistados coincidieron en cuáles discreparon respecto a las variables en torno al mercado objetivo para el cacao. Respecto a la guía de preguntas para el análisis FODA, las respuestas del investigador se ordenaron en una tabla donde se sintetizaron las problemáticas identificadas.

\section{RESULTADOS}

\subsection{Caracterización de las unidades de producción familiar}

De acuerdo a la figura 01 se tiene que en la provincia de Bagua y Utcubamba, el 44\% de los agricultores dispone de hasta 1 hectárea de cultivo destinada a la producción de grano de cacao y el $38 \%$ tiene de 1 a 2 hectáreas, esto significa que las unidades de producción de cacao son pequeñas. El 49\% de productores invierte entre 14000 a 16000 soles/ha, mientras que el 33\% invierten más de 20000 soles/ha de cultivo, la justificación a los resultados

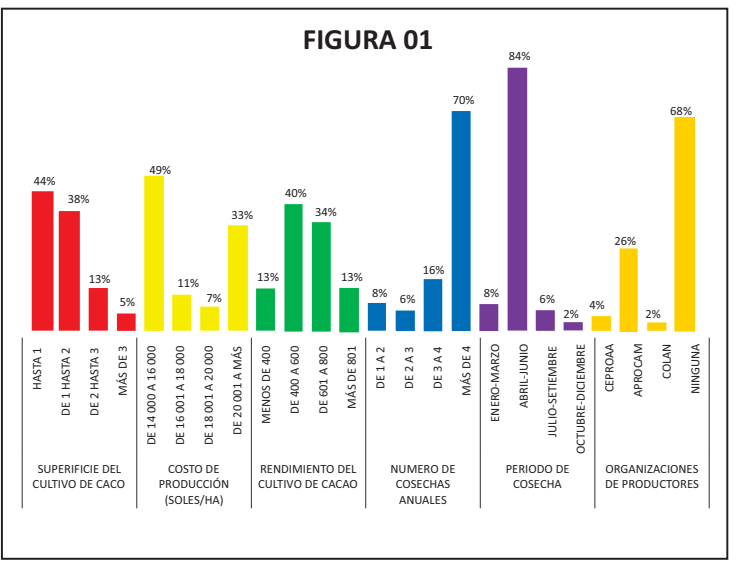


probablemente sea la tecnología empleada y el grado de empleo de insumos y abonos comerciales. Con lo referente al rendimiento del cultivo de cacao, se tiene que el $40 \%$ tiene una producción comprendidas entre $400-600 \mathrm{~kg} / \mathrm{ha}$, y el $34 \%$ con $600-800 \mathrm{~kg} / \mathrm{ha}$, mientras que el $13 \%$ tuvo rendimiento de más de 800 $\mathrm{kg} / \mathrm{ha}$ siendo este el rendimiento promedio nacional. Por otra parte con lo referente de la cantidad de cosechas por año se tiene que la gran mayoría de productores alcanzan más de 4 cosechas por año, representado el 70\%. El 16\% logra de 3 - 4 cosechas y, el 14\% de los productores, menos de $1-3$ cosechas por año. La estacionalidad se encuentra prácticamente definida según el reporte de las encuestas. El $84 \%$ de los productores realizan las cosechas de las bayas de cacao entre los meses de abril a junio. Finalmente con lo referente al tema organizacional, el $4 \%$ pertenece a la Cooperativa Central de Productores Agrarios de Amazonas, el 26\% a la Asociación de Productores y Cafetaleros de Amazonas y el 2\%, perteneció a la Cooperativa Cafetalera La Palma Ltda, la misma que en el último periodo clausuro sus actividades como organización, teniendo como resultado que el $68 \%$ de los productores manifestaron que trabajan de manera individual.

Con lo referente a la figura 02 , se tiene que la asociatividad tiene buena valoración por parte de las familias que producen cacao, ya que el $92 \%$ de la población reconoce que pertenecer a una cooperativa o asociación de productores permite incrementar los ingresos familiares y por tanto, mejorar la calidad de vida. Por otro lado, se observa que el 97\% manifiesta que el cultivo de cacao tiene un efecto positivo en la vida de los productores. Con lo referente a la presentación de venta se tiene que el $53 \%$ se vende como baba y el $39 \%$ como grano crudo. La unidad de medida preferida por el mercado para la venta del

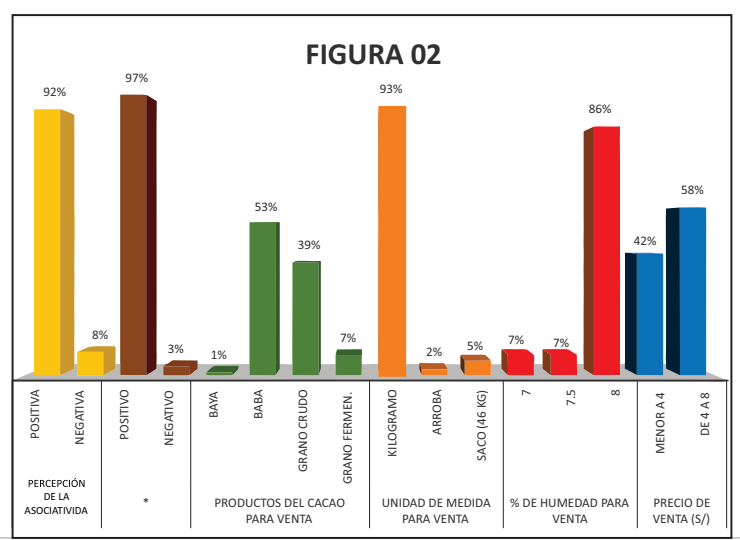

cacao es el kilogramo, así lo demuestra el 93\% de los agricultores. El cacao se vende principalmente con $8 \%$ de humedad, así lo realiza el $86 \%$ de los productores. Finalmente se presenta los precios de venta del cacao en la localidad, que fluctúan según el mes de venta, ya que el $58 \%$ de los agricultores manifiesta que vende el $\mathrm{kg}$ entre $4-8$ soles y el $42 \%$, vende su producto a menos de 4 soles $/ \mathrm{kg}$.

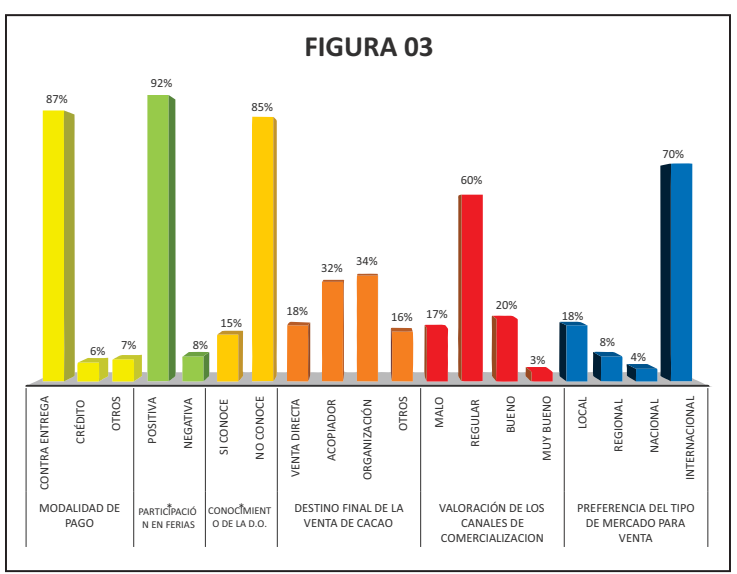

No se han reportado ventas con precio de 8 soles $/ \mathrm{kg}$.

En la figura 03 nos demuestra que la mayor modalidad de pago que se tiene en la comercialización del cacao es la contra entrega, alcanzando el $87 \%$ de los productores participan de esta práctica. El 92\% de los agricultores considera importante la participación en ferias para promocionar su producto y acceder a mercados especializados con mejores precios por kilogramo.

El $85 \%$ de los agricultores del ámbito de la investigación, no tiene conocimiento de la existencia de la denominación de origen Cacao Amazonas Perú. Existe por tanto, debilidades en la socialización y concientización sobre la denominación por parte de las instituciones y organizaciones, mientras que el $15 \%$ de los agricultores reconoce la importancia de la denominación para generar mejoras sustanciales para los agricultores. Existen diferentes destinos para la producción del cacao producido en las provincias de Bagua y Utcubamba, no existiendo una preferencia clara, ya que el 18\% realiza venta directa, el $32 \%$ destina su producción a un acopiador, el 34\% deja su producción para la gestión de la organización de productores y el $16 \%$ prefiere otro destino para su producción. Actualmente, los canales de comercialización son débiles y los productores generalmente consideran que son regulares $(60 \%)$. El $17 \%$ de la población los considera malos. El 20\% cree que los canales son buenos, y apenas el 3\% considera que son muy buenos. Finalmente se tiene que el $70 \%$ de los agricultores considera al mercado 
internacional como el más conveniente para vender cacao. El 18\% prefiere realizarlo en el mercado local, el $8 \%$ en el ámbito regional y el $4 \%$ en el mercado nacional.

\subsection{Diagnóstico comercial de las organizaciones cacaoteras}

El diagnóstico se realizó con base en los resultados de las entrevistas realizadas a los gerentes de las organizaciones de productores y al director general de PRO Cacao Amazonas.

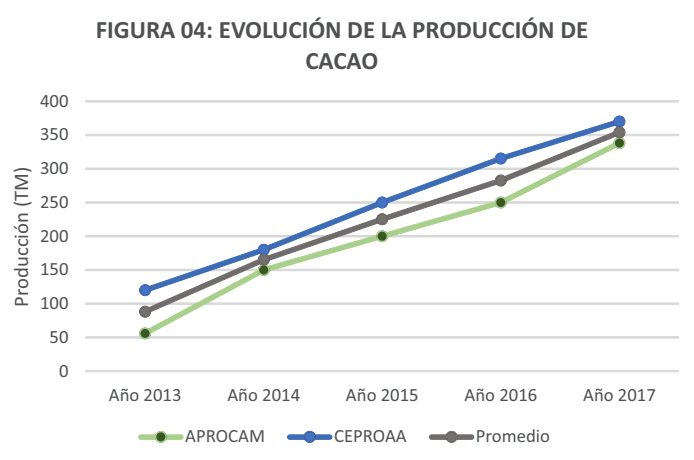

En el último quinquenio la producción de cacao en las provincias de Bagua y Utcubamba, ha tenido un incremento vertiginoso como se muestra en la Figura 04, motivados por el incremento de la demanda nacional e internacional por el producto.

\subsubsection{Análisis FODA}

Tabla 01: Análisis FODA

\subsubsection{Identificación del mercado objetivo}

El análisis ha permitido identificar que los productores tienen como clientes objetivos a empresa $\mathrm{u}$ organizaciones que demandan cacao como materia prima (entre el 95 - $98 \%$ del total de producción). CEPROAA ha logrado obtener un producto con $80 \%$ de fermentación, humedad del 7\%, grano blanco del $8 \%, 0.01 \%$ de impurezas, no existiendo dichas características en el mercado local.

La producción es ínfima si se considera la demanda actual del mercado objetivo, prueba de ello por ejemplo, es que APROCAM sólo logra abastecer el $1 \%$ de lo demandado por ICAM - SPA (Canadá). En el caso de CEPROAA, esta logra abastecer de 20 $30 \%$ de lo requerido por la Empresa Nor Andino.

Ambas organizaciones cuentan con las certificaciones orgánicas, de comercio justo y la denominación de origen, las cuales han permitido mejores precios y mejores mercado en diferentes contextos. Actualmente, el grano de cacao de las provincias de Bagua y Utcubamba ya tiene un pequeño posicionamiento en el mercado internacional debido a la calidad organoléptica, sin embargo, las estrategias para la comercialización deben ser más agresivas, los volúmenes tienen que ser grandes para tener poder en las negociaciones, a través de acuerdos entre las organizaciones.

\begin{tabular}{|c|c|c|}
\hline & Fortalezas & Debilidades \\
\hline $\begin{array}{l}\text { Análisis } \\
\text { interno }\end{array}$ & 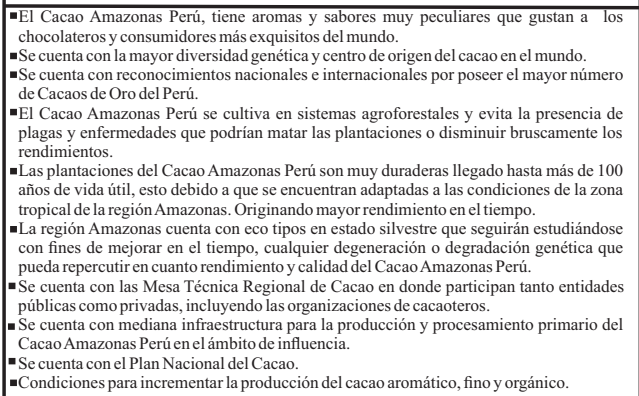 & 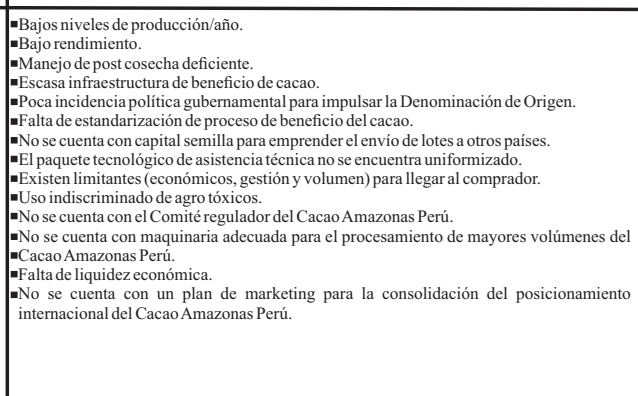 \\
\hline & Oportunidades & Amenazas \\
\hline $\begin{array}{l}\text { Análisis } \\
\text { externo }\end{array}$ & 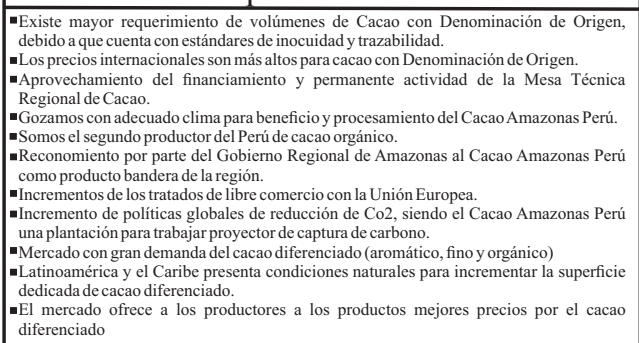 & $\begin{array}{l}\text { - Caída brusca del precio internacional de cacao en los mercados internacionales. } \\
\text { - Que los demás países incrementen su producción de cacao fino de aroma. } \\
\text { - Otras regiones productoras de Cacao del Perú soliciten la denominación de origen. } \\
\text { - Se incremente la producción de cacaos CCN51. } \\
\text { - Que se disminuyan los niveles mínimos permisibles requeridos de contenido de cadmio } \\
\text { de la unión europea. } \\
\text { - Competencia desleal. } \\
\text { - Falta de fidelidad de los socios hacia sus organizaciones. }\end{array}$ \\
\hline
\end{tabular}




\subsection{Modelo de gestión para el posicionamiento comercial del Cacao Amazonas, Perú en mercados nacionales e internacionales}

\subsubsection{Vector de crecimiento}

Con base en la matriz producto - mercado o vector de crecimiento de Ansoff (1957), se ha construido la siguiente matriz (Tabla 02) que contiene las oportunidades de crecimiento identificadas de las organizaciones productoras de cacao en materia comercial. Los insumos que se usaron para construir la matriz fueron la caracterización productiva del cacao, el estado actual de sus productos, la identificación del mercado objetivo y el análisis FODA.

\section{Tabla 02: matriz producto - mercado}

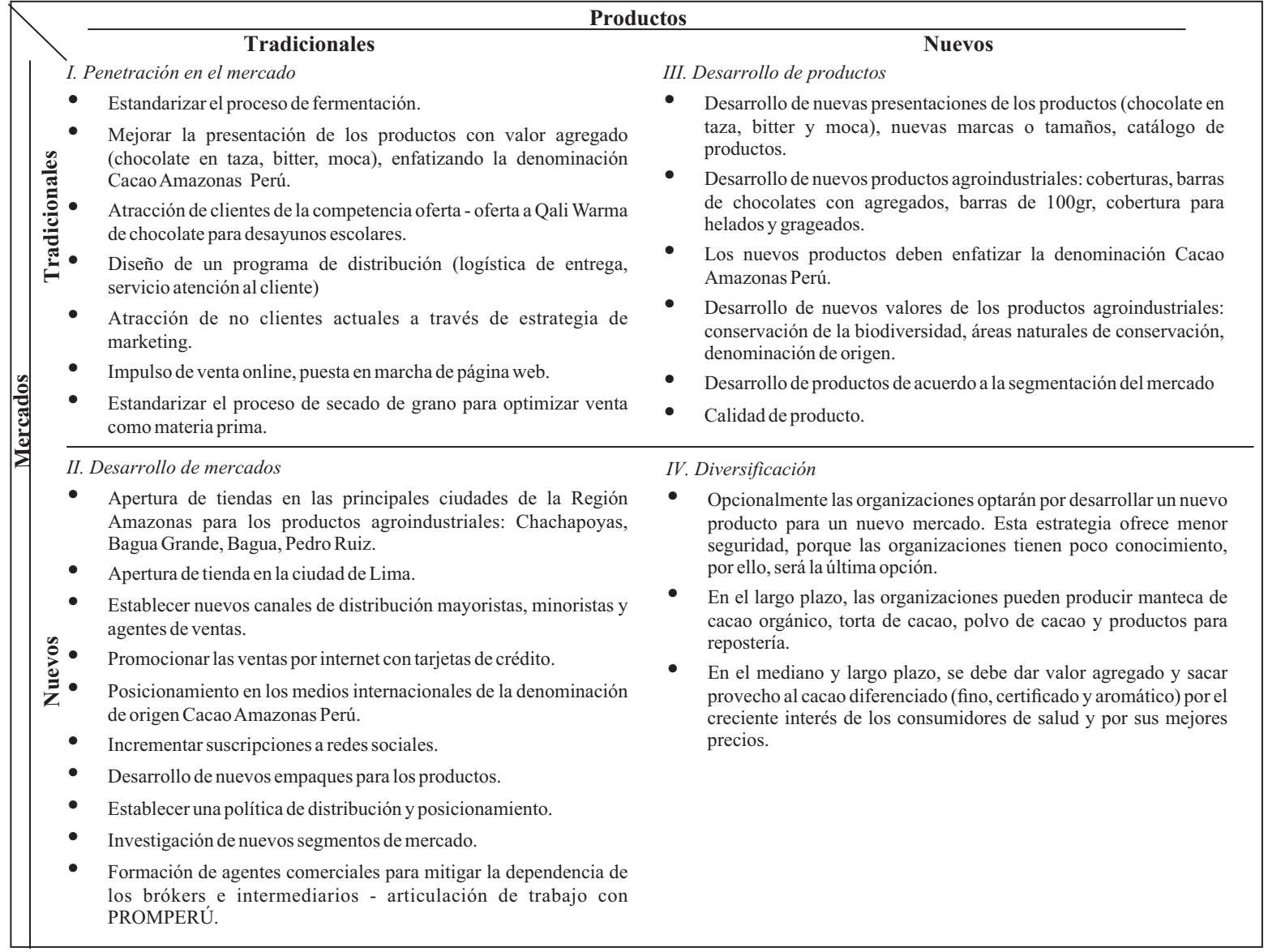

\subsection{2. $\quad$ Estrategias del marketing mix}

Las estrategias presentadas para la diversas combinaciones de productos y mercados serán fortalecidas con estrategias del marketing mix en función de las 7Ps (CIM, 2015).

En la tabla 03 se muestra un esbozo que representa los lineamientos básicos (acciones), que deben abordarse en un plan de marketing para el posicionamiento, en sus distintos niveles, de los productos del Cacao Amazonas Perú.

Una combinación de estas acciones puede ser utilizada por las organizaciones para emprender sus estrategias en los distintos niveles del posicionamiento a nivel internacional, como se muestra a continuación:

Tabla 03: Plan de Marketing

\begin{tabular}{|l|l|}
\hline Elementos & \multicolumn{1}{c|}{ Acciones a desarrollar } \\
\hline 1. Producto & $\begin{array}{l}\text { Mejorar la presentación (envases, etiquetas, logos) de los productos tradicionales; desarrollo de nuevos productos enfatizandola } \\
\text { denominación de origen (coberturas, barras de chocolates con agregados, barras de 100gr, cobertura para helado, entre otros). } \\
\text { Estandarización de los procesos de secado y fermentación. Desarrollo de productos nuevos para mercados nuevos } \\
\text { (industrias)(manteca de cacao orgánico, polvo de cacao, entre otros). Gestión de permisos para comercializar la denominación } \\
\text { Cacao Amazonas Perú. Compra de equipos y maquinaria. Puesta en valor de las certificaciones obtenidas Incrementar la } \\
\text { producciónde cacao diferenciado aromático, fino yorgánico }\end{array}$ \\
\hline
\end{tabular}




\begin{tabular}{|c|c|}
\hline 2. Precio & $\begin{array}{l}\text { Reducir los costos de producción; generar ventas por internet, servicios de atención al cliente sin costo adicional; empoderamiento } \\
\text { de la denominación origen durante las etapas de negación y colocación del grano de cacao; tarifas segmentadas. }\end{array}$ \\
\hline 3. Plaza & $\begin{array}{l}\text { Generar espacios apropiados para las ventas; desarrollo del sistema web para ventas por internet, mostrador referencial de los } \\
\text { productos, correo electrónico corporativo, formación de agentes de venta, desarrollo de canales de distribución nuevos } \\
\text { (mayoristas, minoritas, agentes) }\end{array}$ \\
\hline 4. Promoción & $\begin{array}{l}\text { Gestión de redes sociales; estrategias para incrementar las suscripciones por internet; venta personal; panfletos; gestión de correos } \\
\text { electrónicos; boletines informativos; sistema de consulta-respuesta online; afiches; flyers; estrategia de volanteo; exhibición de } \\
\text { productos; anuncios radiales, televisivos. }\end{array}$ \\
\hline $\begin{array}{c}\text { 5. Pleople } \\
\text { (Gente, en español) }\end{array}$ & Capacitar agentes; fortalecimiento de las capacidades del personas a través de talleres y asistencia técnica. \\
\hline $\begin{array}{l}\text { 6. Proceso / } \\
\text { participantes }\end{array}$ & Asistencia telefónica o en línea; servicio online a dudas o preguntas. \\
\hline $\begin{array}{l}\text { 7. Physical evidence } \\
\text { (evidencia física, en } \\
\text { español) }\end{array}$ & $\begin{array}{l}\text { Decorar los ambientes administrativos y productivos de las organizaciones; elaboración de manuales; mejoramiento de las } \\
\text { instalaciones, gestión de la imagen de los sitios web. }\end{array}$ \\
\hline
\end{tabular}

\subsubsection{Sistematización de las estrategias de posicionamiento}

Para sistematizar las estrategias de posicionamiento de la denominación de origen, se ha divido, conforme a Lazo (2006), en cuatro categorías: a) posicionamiento de marca o producto, b) posicionamiento de sector clúster, c) posicionamiento país y d) posicionamiento global. La desagregación de las estrategias se realizó porque se trata de un producto agrícola en un país en vías de desarrollo. El primer nivel de posicionamiento se ha elaborado con más detalle, por estar al alcance de las organizaciones de productores. Los tres últimos niveles de posicionamiento dependen de los contextos nacionales e internacionales, por lo que se presentó un esquema conciso.

\section{Tabla 04: Sistematización de las estrategias de posicionamiento}

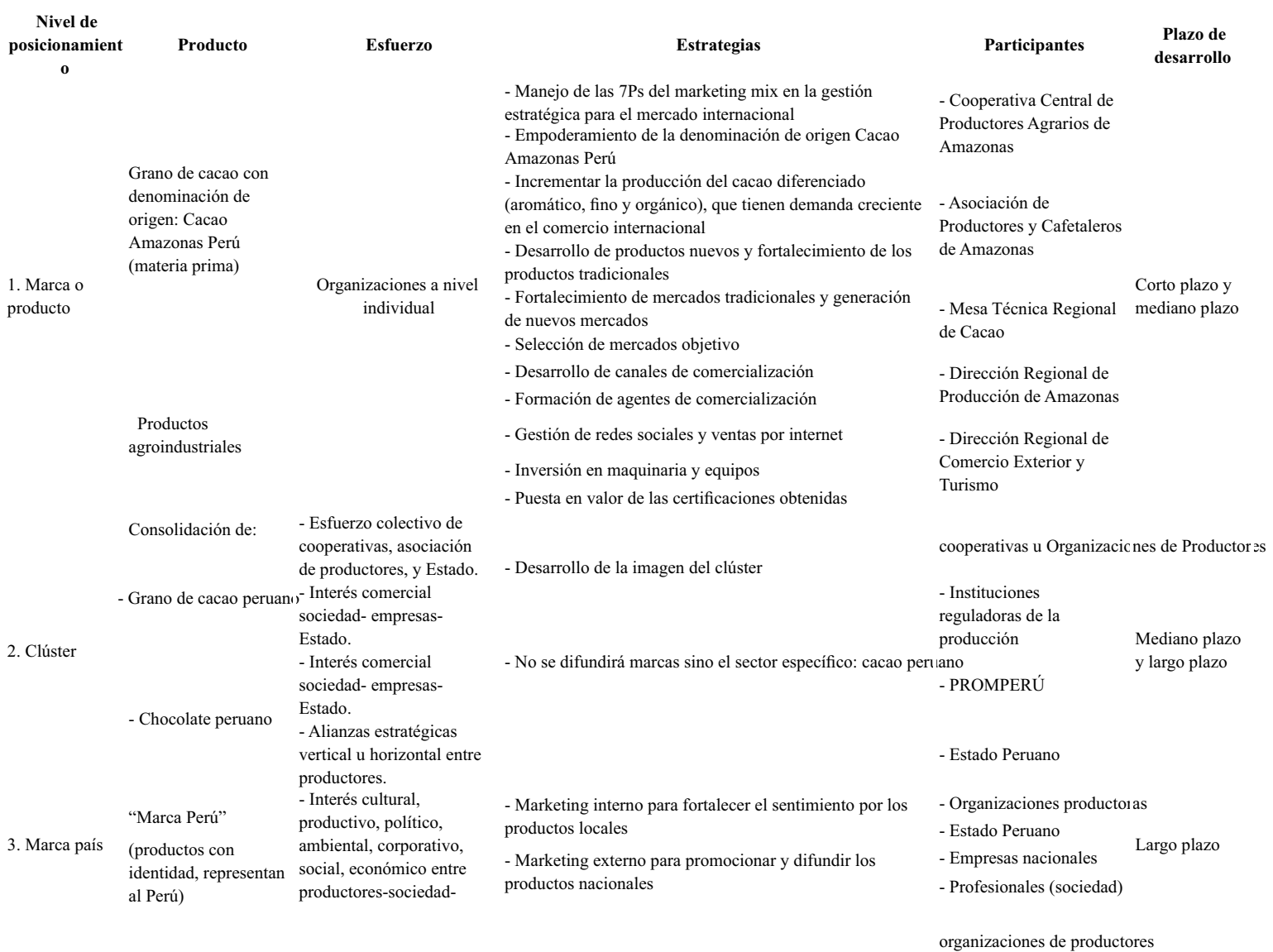




\section{DISCUSIÓN}

Tal como se esperaba, los rendimientos del cultivo de cacao de las organizaciones productoras son cercanos a los reportes nacionales e internacionales. La investigación ha reportado rendimientos en Bagua y Utcubamba entre los $400-600 \mathrm{~kg} / \mathrm{ha}$. La región Amazonas tiene un rendimiento que oscila entre $700-900 \mathrm{~kg} / \mathrm{ha}$ (Rosa-Pérez et al., 2015). En el ámbito internacional, Arvelo et al. (2016) reporta un promedio mundial de $400 \mathrm{~kg} / \mathrm{ha}$ y el promedio de Perú es de $610 \mathrm{~kg} / \mathrm{ha}$ en el año 2012. En el año 2014 el promedio mundial fue de $426.5 \mathrm{~kg} / \mathrm{ha}$ (ToledoHernández, Wanger, \& Tscharntke, 2017).

La producción de cacao en Bagua y Utcubamba se realiza en unidades familiares, realidad similar a la de América Latina y el Caribe, donde el $90 \%$ de la producción se encuentra en pequeños y medianos agricultores. En promedio, cada familia dispone de 1.37 hectáreas de cultivo en Bagua y Utcubamba, por debajo del promedio nacional de 2.17 hectáreas por familia, y de los principales productores americanos: Ecuador con 4.47 hectáreas y República Dominicana con 4.19 hectáreas (Arvelo et al., 2016).

A diferencia del cacao convencional, la demanda de cacao diferenciado (fino, certificado y aromático) se proyecta a crecer puesto que la industria del chocolate responde al interés de los demandantes de productos saludables, de origen diferenciado y amigables con la biodiversidad (ECLAC et al., 2017). Sumado a ello, la etiqueta de comercio justo permitirá ingresos dignos para los agricultores y una participación en el largo plazo con productos de calidad para los compradores (Beg, Sameer, Jan, \& Bashir, 2017).

Las organizaciones evaluadas de Bagua y Utcubamba producen cacao fino, certificado y con denominación de origen. Esto les sitúa en condición privilegiada y de provecho en el contexto del comercio mundial, ya que se paga mucho más por ellos, además, los países Latinoamericanos y del Caribe presentan condiciones idóneas para explotar este producto. América Latina es líder en la producción de cacao diferenciado (cacao fino, aromático y certificado), donde Ecuador es principal productor de cacao fino y aromático con 55\% del mercado, luego están Papúa Nueva Guinea con 14\%, República Dominicana con 11\% y Perú con $9 \%$. El cacao orgánico es producido principalmente por Perú, Costa Rica, Madagascar, Tanzania, Uganda,
Belice, Bolivia, Brasil, República Dominicana, El Salvador, México, Nicaragua, Panamá y Venezuela (Arvelo et al., 2016).

Las estrategias identificadas para el posicionamiento comercial del cacao, con base en la denominación de origen para generar nuevos productos, nuevos mercados y la diversificación, están en consonancia con las tendencias del mercado internacional del cacao: casi la mitad del total del cacao importado por consumidores, es cacao con valor agregado, la otra mitad es en grano. Europa es el principal consumidor de estos productos, con $62 \%$ de las importaciones, debido a que sus consumidores se han vuelto más conscientes de los beneficios para la salud de comer chocolate producido con cacao aromático y certificado (Dand, 2011a). Según Arvelo et al. (2016), Europa consume el 58.6\% de la producción mundial, y su población tiene una tasa de consumo per cápita de $2.27 \mathrm{~kg} /$ año, muy superior al promedio mundial $0.64 \mathrm{~kg} / \mathrm{año}$. El resto, es consumido por le América con $19.3 \%$, Asia y Oceanía con $21.7 \%$ y África con $0.6 \%$. Los tres principales factores que contribuyen a que Europa sea el principal consumidor son: el poder adquisitivo, el clima y la cultura (Dand, 2011a)

Si bien, una estrategia para el posicionamiento es la reducción del papel de los intermediarios, esta se debe entender como una estrategia de largo plazo. Actualmente los intermediarios juegan un rol importante, ya que facilitan el comercio mundial del cacao. Las razones por las cuales pocos productores independientes pueden comerciar directamente con los usuarios finales son: no siempre un productor que desea vender encontrará un comprador que quiera comprar, igualmente, un comprador no siempre encontrará un productor preparado para vender en un día particular, es por ello, que el mercado desarrolló agentes de servicios capaces de atender las necesidades de productores del Hemisferio Sur y de compradores en el Hemisferio Norte; sin ellos los precios subirían y bajarían bruscamente, dan alivio a los compradores ofreciendo materia prima en cualquier época del año y, garantizan compradores a los productores, aunque también reducen su ganancias (Dand, 2011b). Eliminar los intermediarios, por tanto, requiere inversión por parte de las organizaciones y de la cooperación de las instituciones reguladoras, para la formación de 
agentes muy capacitados en un escenario de mediano y largo plazo.

Finalmente, la tendencia internacional de la demanda de cacao presenta para la región Amazonas, un escenario prominente en el mediano plazo. Actualmente el mercado del cacao crece a un ritmo de $3 \%$ en todo el mundo $\mathrm{y}$, según las proyecciones, la demanda mundial está en continuo crecimiento y puede llegar a incrementarse hasta $30 \%$ para 2020 (Beg et al., 2017). Esto implica que las estrategias de posicionamiento deben estar acompañadas de estrategias para incrementar las superficies de cultivo.

\section{CONCLUSIONES}

En el último quinquenio las organizaciones han incrementado vertiginosamente su producción, la cual se ha cuadriplicado, pasando de 88 TM en el año 2013 a 354 TM en 2017. El rendimiento varia en promedio entre $400-600 \mathrm{~kg}$ de cacao $/ \mathrm{ha}$.

La denominación de origen Cacao Amazonas Perú, pese a su importancia para genera valor del producto y mejores ingresos para los productores, es de poco conocimiento por los agricultores. Apenas el 15\% conoce su significado y reconoce su importancia socioeconómica. El cacao producido se vende casi exclusivamente en el ámbito internacional, los principales países compradores son: Francia, Italia, Canadá, Suiza y Ecuador.

Los niveles productivos son pequeños, por ejemplo APROCAM sólo logra abastecer el $1 \%$ de lo demandado por ICAM - SPA (Canadá) y CEPROAA del $20-30 \%$ de lo requerido por la Empresa Nor Andino. El volumen actual de ventas varía entre 350 $400 \mathrm{tm} / \mathrm{año}$, se vende con frecuencia de $01 \mathrm{vez} / \mathrm{mes}$ o quincenalmente.

Las organizaciones estudiadas, han obtenido tres certificaciones de sostenibilidad: certificación orgánica, de comercio justo y la denominación de origen Cacao Amazonas Perú. Sin embargo, esta última no se aprovecha porque no cuentan con comité regular para emisión de certificados.

El principal producto es el cacao en grano para venta internacional $(95-98 \%$ de la producción total). El resto se utiliza para producir productos agroindustriales para el mercado local.

Para lograr el posicionamiento del cacao con denominación de origen en el mercado internacional se han identificado tres caminos: a) penetración en el mercado, b) desarrollo de mercados, c) desarrollos de productos y d) diversificación.
La penetración en el mercado consiste en incrementar el consumo de los productos actuales en con los mercados actuales (clientes). El desarrollo de mercados tiene la finalidad de vender productos actuales en mercados nuevos, el desarrollo de productos tiene la finalidad de vender nuevos productos en los mercados actuales. Asimismo, desarrollar nuevos valores para la imagen corporativa de las organizaciones como: conservación de la biodiversidad, promoción de la denominación de origen Cacao Amazonas, Perú.

La estrategia de diversificación nuevos productos para nuevos mercados, debe ser la última estrategia en ser abordada ya que ofrece menos seguridad y mayor incertidumbre, y además, debe hacerse en el largo plazo. Sumado a las tres estrategias, las organizaciones deberán complementarlas con estrategias del marketing mix con base en las 7 Ps en el contexto del mercado internacional.

Finalmente, como también recomienda Arvelo et al. (2016), las organizaciones de Utcubamba y Bagua deben aumentar su esfuerzos en producir cacao diferenciado (aromático, fino y orgánico), puesto que, las tendencias del mercado demuestra altísima demanda de cacao diferenciado, se paga mucho más por ellos, a eso se agregar que, los países Latinoamericanos y del Caribe tienen las condiciones climáticas y geográficas idóneas para explotar este producto.

\section{VI .REFERENCIAS}

Ansoff, H. I. (1957). Strategies for Diversification. Harvard Business Review, 35(5), 113-124. $\mathrm{R}$ e $\mathrm{t}$ r i e v e d f r o m https:/www.casrilanka.com/casl/images/st ories/2017/2017_pdfs/sab_portal/course_m aterial/strategies_for_diversification.pdf

Arvelo, M. A., Delgado, T., Maroto, S., Rivera, J., Higuera, I., \& Navarro, A. (2016). Estado actual sobre la producción y el comercio del cacao en América. San José, Costa Rica: Instituto Interamericano de Cooperación para la Agricultura (IICA). Retrieved from https://www.iica.int/sites/default/files/publ ications/files/2017/BVE17048806e.pdf

Beg, M. S., Sameer, A., Jan, K., \& Bashir, K. (2017). Status, supply chain and processing of cocoa - A review. Trends in Food Science \& Te c hno log y, $66,108-116$. https://doi.org/10.1016/j.tifs.2017.06.007

Blare, T., \& Useche, P. (2013). Competing objectives of smallholder producers in developing 
countries: examining cacao production in Northern Ecuador. Environmental Economics, 4(1), 71-79. Retrieved from http://www.researchgate.net/profile/Trent Blare/publication/269278023_Competing_ objectives_of_smallholder_producers_in_d eveloping_countries_examining_cacao_pr oduction_in_Northern_Ecuador/links/5485 e1ab0cf289302e28f504.pdf

CIM, T. C. I. of M. (2015). Marketing and the 7Ps: A brief summary of marketing and how it work. The Chartered Institute of Marketing ( $\left.\begin{array}{ccc}\mathrm{C} & \mathrm{I} & \mathrm{M}\end{array}\right)$. https://doi.org/10.1177/0002764295038004 007

Colovic, G. (2012). Strategic management. In G. Colovic (Ed.), Strategic Management in the Garment Industry ( p p . 1-13). https://doi.org/10.1533/9780857095855.1

Dand, R. (2011a). International consumption and stocks of cocoa. In The International Cocoa Trade (pp. 190-218). London: Woodhead Publishing. https://doi.org/10.1016/B9780-85709-125-3.50007-0

Dand, R. (2011b). The physical market in the international cocoa trade. In The International Cocoa Trade (pp. 94-118). London: Woodhead Publishing. https://doi.org/10.1016/B978-0-85709-1253.50004-5

De Saez, E. (2002). Marketing concepts for libraries and information services. London: Facet Publishing.

De Souza, P. A., Moreira, L. F., Sarmento, D. H. A., \& da Costa, F. B. (2018). Cacao - Theobroma cacao. In S. Rodrigues, E. De Olivera Silva, $\&$ E. Sousa de Brito (Eds.), Exotic Fruits (Vol. 3, pp. 69-76). Elsevier. https://doi.org/10.1016/B978-0-12-8031384.00010-1

ECLAC, E. C. for L. A. and the C., FAO, F. and A. O. of the U. N., \& IICA, I.-A. I. for C. on A. (2017). The Outlook for Agriculture and Rural Development in the Americas: A Perspective on Latin America and the Caribbean 2017-2018. (M. García-Winder \& H. Chavarría, Eds.). San José, Costa Rica. R e t r i e v e d f r o m https://repositorio.cepal.org/handle/11362/4 2282
Fisher, P. H., \& Pride, M. M. (2006). Blueprint for your library marketing plan: A guide to help you survive and thrive. Chicago: American Library Association.

Garoufallou, E., Siatri, R., Zafeiriou, G., \& Balampanidou, E. (2013). The use of marketing concepts in library services: A literature review. Library Review, 62(4/5), 312-334. https://doi.org/10.1108/LR-062012-0061

Gayi, S. K. (2014). Improving the efficiency and transparency of the Cocoa Global Value Chain (GVC). Market structure and potential impacts on smallholder farmers. Amsterdam.

Humphrey, A. S. (1974). Getting Management Commitment to Planning - A New Approach. Long Range Planning, 7(1), 45-51. https://doi.org/10.1016/00246301(74)90078-8

Kotler, P., \& Armstrong, G. (2012). Principles of Marketing. (E. Svendsen, M. Sabella, J. Leale, \& J. Keefe, Eds.), Principles of Marketing (14th ed.). New Jersey: Prentice Hall. https://doi.org/10.2307/1250103

Kotler, P., \& Keller, K. L. (2012). Marketing Management. (E. Svendsen, E. Adams, M. Sabella, \& J. Leale, Eds.), Marketing Management (14th ed.). New Jersey: P r e $n$ t i c e $\mathrm{H}$ a 11 . https://doi.org/10.1080/0891176090302255 6

Lazo, L. (2006). Niveles de posicionamiento de marca a nivel internacional. Contabilidad y Negocios, 1(2), 36-40. Retrieved from http://www.redalyc.org/html/2816/2816217 66008/

Martinet, A. C. (2010). Strategic planning, strategic management, strategic foresight: The seminal work of $\mathrm{H}$. Igor Ansoff. Technological Forecasting and Social Change, 77 (9), $1485-1487$. https://doi.org/10.1016/j.techfore.2010.06.0 24

Nair, K. P. P. (2010). Cocoa (Theobroma cacao L.). In The Agronomy and Economy of Important Tree Crops of the Developing World (pp. $131-180)$. E $1 \mathrm{~s}$ e vi e r. https://doi.org/10.1016/B978-0-12-3846778.00005-9

Rosa-Pérez, L., Canales, M., \& Santoyo, T. (2015). 
Análisis de la cadena de valor del Cacao Fino de Aroma en la Región Amazonas. Lima, Perú.

Summers, J., Gardiner, M., Lamb, C. W., Hair, J. F., \& McDaniel, C. (2005). Essentials of marketing (2nd ed.). South Melbourne, Victoria: Nelson Australia Pty Limited.

Sun, S. (2009). An Analysis on the Conditions and Methods of Market Segmentation. International Journal of Business and $\mathrm{M}$ a n a g e m e n t, 4 ( 2), 63-70. https://doi.org/10.5539/ijbm.v4n2p63

Toledo-Hernández, M., Wanger, T. C., \& Tscharntke, T. (2017). Agriculture, Ecosystems and Environment Neglected pollinators : Can enhanced pollination services improve cocoa yields? A review. Agriculture, Ecosystems and Environment, 247(August $\left.\begin{array}{llll}2 & 0 & 1 & 6\end{array}\right), \quad 137-148$. https://doi.org/10.1016/j.agee.2017.05.021

Welch, L. (2006). The other 51 weeks of the year: A marketing handbook for librarians (Rev. ed.). Wagga Wagga, NSW: Centre for Information Studies, Charles Sturt University 